\title{
Effect of Adiabaticity on Electron Dynamics in Zinc Myoglobin
}

\author{
Byung Moon Cho, ${ }^{\ddagger}$ Ross C. Walker, ${ }^{\dagger,}$ Halima Amer, ${ }^{\prime \prime}$ Ian Mercer, ${ }^{\ddagger}$ David R. Klug, ${ }^{\ddagger}$ and \\ Ian R. Gould*, \\ Biological and Biophysical Chemistry Group and Molecular Dynamics Group, Department of Chemistry, \\ Imperial College London, London, U.K.
}

Received: July 2, 2004; In Final Form: November 24, 2004

\begin{abstract}
Electron-vibration coupling in zinc substituted myoglobin has been calculated using a quantum mechanical/ molecular mechanical method. The methodology has been tested by a direct comparison of the calculated optical observables, the steady-state optical spectra and three-pulse-photon-echo-peak-shift (3PEPS) function, to those experimentally measured showing a qualitative agreement. A range of experiments and calculations were performed to explain the discrepancies, which lead to the conclusion that the discrepancy originates from adiabatic coupling of the two nearly degenerate electronic transitions.
\end{abstract}

\section{Introduction}

Central to an understanding of chemical dynamics in condensed phases is the knowledge of how the energy fluctuation of a solvated macromolecule is influenced by its environment. For example, in nonadiabatic electron-transfer theory, the origin and the curvature of the quadratic free energy surfaces are due to thermally induced fluctuations. ${ }^{1}$ These fluctuations arise from electron-vibration coupling.

For a chromophore bound to a protein, this coupling of the electronic energy levels of the chromophore to vibrations allows energy to be exchanged between the chromophore and its environment (protein bulk and solvent). Direct manifestations of such electron-vibration coupling are the Stokes shift and linebroadening.

It is not possible to deduce from the steady-state spectra alone the time scales of the individual coupled interactions. This is because steady-state spectra can only provide ensemble averaged information. For instance, the width of an absorption spectrum could result from a large number of slowly interconverting structures with different energy (inhomogeneous broadening), from fast processes that randomize the energy (homogeneous broadening), from unresolved vibrational progressions or from a combination of all three. In addition, the meaning of the term "fast" or "slow" becomes unclear when the time scale of the measurement is similar to that of the fluctuation. To get the time-scale information, time-resolved nonlinear optical methods such as three-pulse-photon-echo-peak-shift (3PEPS) measurements are necessary.

We have previously used our recently developed quantum mechanical/molecular mechanical (QM/MM) method, for studying equilibrium fluctuations in optical excitations, to calculate electron-vibration coupling in enzymes and proteins. ${ }^{2,3}$ These previous works showed that our QM/MM methodology can recover the fluorescence Stokes shift and absorption width to

* To whom correspondence should be addressed. E-mail: i.gould@ imperial.ac.uk.

Biological and Biophysical Chemistry Group.

Molecular Dynamics Group.

$\S$ Current address: Department of Molecular Biology TPC15, The Scripps Research Institute, 10550 N. Torrey Pines Road, La Jolla, CA 92037.

"Current address: Lawrence Livermore National Lab, 7000 East Ave., Livermore, CA 94550-9234. better than $20 \%$ of the experimental value. In this work, we apply the same method to calculate the electron-vibration coupling in zinc porphyrin IX substituted myoglobin. Strictly speaking, the Stokes shift includes contribution from FranckCondon progression which is not well-represented in the classical MD simulations when the frequencies are much higher than $\mathrm{kT}$, and vibrational sidebands do not therefore show up well in the calculated spectra. We, however, are interested in the contribution of the lower frequencies which cause line broadening. To remove any ambiguity we use the term peak shift to represent the shift between absorption and emission maxima to distinguish this from the total Stokes shift.

The peak shift in zinc protoporphyrin IX $(\mathrm{ZnP})$ bound to myoglobin (ZnP-MB) measures $\sim 0.007 \mathrm{eV}$. We performed classical molecular dynamic calculations on $\mathrm{ZnP}-\mathrm{MB}$, using the AMBER 6.0 suite of molecular dynamics programs. ${ }^{4} \mathrm{We}$ subsequently used the resulting trajectory to calculate fluctuating ground- to excited-state energy gaps for $\mathrm{ZnP}$ within the charge field of the protein and solvent. From the time-dependent energy gap, we determined the auto-correlation function of the fluctuating energy gap which is the central quantity that allows us to calculate the optical absorption and emission spectra, via optical response theory. The methodology was tested by a direct comparison of the calculated spectra with those experimentally measured.

Since the time-ordering is maintained during the sampling of the phase-space, the time-dependent information is contained within the fluctuating energy gap trace. The time-varying optical observable, such as the 3PEPS function, can be calculated via the autocorrelation function and reveals the extent of homogeneous or inhomogeneous broadening. Simulation of the 3PEPS function tests whether the QM/MM methodology can correctly account for the dynamics. ZnP-MB conveniently absorbs in the visible region. This made it possible to carry out a 3PEPS measurement for a direct comparison to the calculation.

\section{Methodology}

Molecular Dynamics Protocol. The starting structure for zinc-myoglobin was obtained from the horse-heart iron-myoglobin crystal structure determined by Maurus et al. ${ }^{5}$ (RCSB Protein Data Bank 1WLA). The Fe atom was replaced with 
$\mathrm{Zn}$, to give zinc-myoglobin. No bonded interactions between the $\mathrm{Zn}$ atom and the heme unit were included, and so the zinc moiety was effectively modeled as a $\mathrm{Zn}^{2+}$ ion.

The extracted coordinates were prepared for the molecular dynamics simulations using the xLEaP module ${ }^{6}$ which forms part of the AMBER 6.0 suite of Molecular Dynamics programs. ${ }^{4}$ Due to the lack of experimental data for the histidine tautomeric states in horse-heart myoglobin, the states for the sperm-whale variant of myoglobin were used. These have been well characterized by a combination of NMR, $\mathrm{p} K$ and modeling studies. ${ }^{7,8}$ Histidine residues 12, 48, 64, 81, 82, 97, 113, 116 and 119 were therefore defined as being protonated on the $\epsilon$ nitrogen (HIE) and residues 24, 36 and 93 as protonated on the $\delta$ nitrogen (HID). Parameters for heme are not available in the standard Amber force field ${ }^{9}$ and so the parameters of Giammona ${ }^{10}$ were used.

The hydrogen atoms, not present in the crystal structure, were added at idealized bond lengths and angles as necessary to fulfill valence requirements. The system was then neutralized by the addition of two $\mathrm{Na}^{+}$ions distributed in a shell around the zincmyoglobin using a Coulombic potential on a $1 \AA$ grid. The whole system was then solvated using the TIP $3 \mathrm{P}^{11}$ water model by placing it in a box of dimensions $66.2 \AA \times 60.9 \AA \times 62.3$ $\AA$ containing 5638 water molecules, to yield a system of 19404 atoms with an average density of $0.788 \mathrm{~g} \mathrm{~cm}^{-3}$.

Atom centered point charges for the nonstandard $\mathrm{Zn}$-heme unit were derived for the ground state in accordance with the RESP $^{12}$ method for the Amber force field. ${ }^{9}$ The heme unit hydrogen atoms were optimized using Hartree-Fock ${ }^{13}$ STO$3 \mathrm{G}^{14}$ calculations implemented within the Gaussian 98 package. ${ }^{15}$ All heavy atoms were kept fixed during these calculations. The electrostatic potential, required for the RESP fitting procedure, was obtained using a single point HF calculation on the hydrogen optimized zinc-heme structure. The Ahlrichs pVDZ basis set ${ }^{16}$ was used for the zinc atom, while the SV $6-31 G^{* 17}$ basis set was used for all other atoms. The derived atom centered point charges are given in Table 1 .

A molecular dynamics simulation of the zinc-myoglobin in water system was performed using similar methodology to our previous work ${ }^{1,3}$ on equilibrium dynamics. The program Sander, incorporated within the AMBER $6.0^{4}$ suite of molecular dynamics programs, was used to carry out a classical MD simulation of the solvated zinc-myoglobin system. The system was first subjected to 200 steps of steepest descent followed by 800 steps of conjugate gradient minimization in order to alleviate incorrect van der Waals contacts created by hydrogenation and solvation of the system. A residue based nonbonded cutoff of $9 \AA$ was used for this and all subsequent simulations. The system was then subjected to 30 ps of slow heating from 0 to $300 \mathrm{~K}$ following the method of Berendsen ${ }^{18}$ to control the temperature. After slow heating the system was equilibrated at $300 \mathrm{~K}$ for $100 \mathrm{ps}$. Equilibration was deemed to have been successfully obtained when the Root Mean Square Deviation (RMSD) of the protein $\alpha$ carbons was reasonably small $(<1.4$ $\AA$ ) and both the RMSD and classical energies of the system fluctuated by less than $10 \%$ over a time scale of approximately 20 ps. An energy gap production run was then performed for the subsequent 22 ps with the complete system coordinates being recorded every $4 \mathrm{fs}$, resulting in a trajectory of 5,500 coordinate sets.

In all stages of the MD simulation constant pressure periodic boundary conditions using the Particle Mesh Ewald method ${ }^{19}$
TABLE 1: Atomic Partial Charges Used for the Porphyrin Unit of Zinc-Substituted Myoglobin, Fitted to the Calculated Molecular Electrostatic Potentials According to the RESP Method of Bayly et al. ${ }^{12} a$

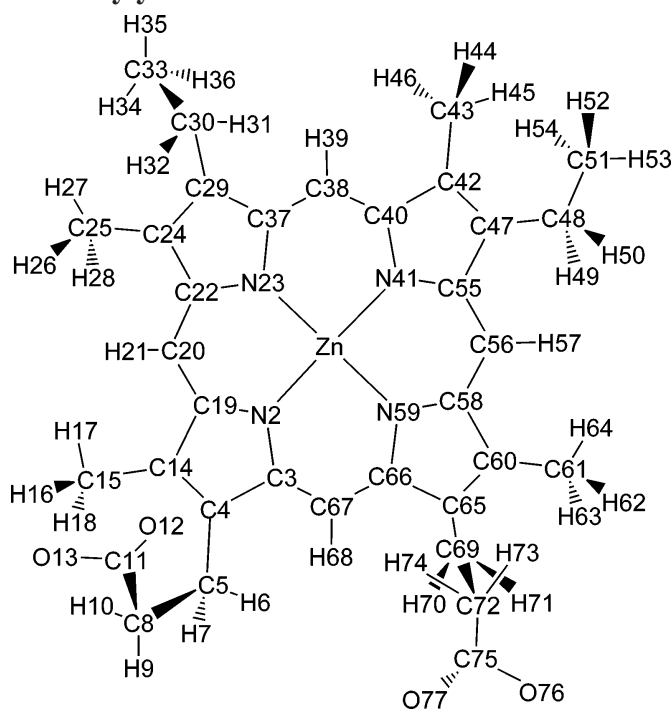

\begin{tabular}{|c|c|c|c|c|c|c|c|c|}
\hline \multicolumn{9}{|c|}{$\mathrm{Zn}-\mathrm{Mb}$ porphyrin group atom types and charges } \\
\hline atom & type & charge & atom & type & charge & atom & type & charge \\
\hline $\mathrm{Zn}$ & ZN & 1.01669 & $\mathrm{H} 27$ & $\mathrm{HC}$ & 0.06177 & H53 & $\mathrm{HC}$ & 0.00019 \\
\hline $\mathrm{N} 2$ & NP & -0.49031 & $\mathrm{H} 28$ & $\mathrm{HC}$ & 0.04681 & H54 & $\mathrm{HC}$ & 0.02801 \\
\hline $\mathrm{C} 3$ & $\mathrm{CC}$ & 0.14780 & $\mathrm{C} 29$ & $\mathrm{CB}$ & -0.04446 & C55 & $\mathrm{CC}$ & -0.02386 \\
\hline $\mathrm{C} 4$ & CB & -0.05709 & C30 & CT & 0.01364 & C56 & CD & -0.04352 \\
\hline C5 & CT & -0.00517 & H31 & $\mathrm{HC}$ & 0.01297 & H57 & $\mathrm{HC}$ & 0.07141 \\
\hline H6 & $\mathrm{HC}$ & 0.00306 & H32 & $\mathrm{HC}$ & 0.00814 & C58 & $\mathrm{CC}$ & 0.01204 \\
\hline $\mathrm{H} 7$ & $\mathrm{HC}$ & 0.00306 & C33 & CT & -0.01514 & N59 & NO & -0.43114 \\
\hline C8 & $\mathrm{CT}$ & 0.06110 & H34 & $\mathrm{HC}$ & -0.00262 & C60 & $\mathrm{CB}$ & 0.04520 \\
\hline H9 & $\mathrm{HC}$ & -0.02673 & H35 & $\mathrm{HC}$ & -0.00262 & C61 & CT & -0.13838 \\
\hline H10 & $\mathrm{HC}$ & -0.07037 & H36 & $\mathrm{HC}$ & -0.00262 & H62 & $\mathrm{HC}$ & 0.03987 \\
\hline $\mathrm{C} 11$ & $\mathrm{C}$ & 0.70741 & C37 & $\mathrm{CC}$ & -0.02130 & H63 & $\mathrm{HC}$ & 0.02703 \\
\hline $\mathrm{O} 12$ & $\mathrm{O} 2$ & -0.78807 & C38 & $\mathrm{CD}$ & -0.14138 & H64 & $\mathrm{HC}$ & 0.07299 \\
\hline $\mathrm{O} 13$ & $\mathrm{O} 2$ & -0.78849 & H39 & $\mathrm{HC}$ & 0.14707 & C65 & $\mathrm{CB}$ & -0.02777 \\
\hline C14 & $\mathrm{CB}$ & 0.04791 & $\mathrm{C} 40$ & $\mathrm{CC}$ & 0.00187 & C66 & $\mathrm{CC}$ & 0.08495 \\
\hline $\mathrm{C} 15$ & CT & -0.11610 & N41 & NP & -0.38759 & C67 & CD & 0.03505 \\
\hline H16 & $\mathrm{HC}$ & 0.04176 & $\mathrm{C} 42$ & $\mathrm{CB}$ & & H68 & $\mathrm{HC}$ & 0.17036 \\
\hline H17 & $\mathrm{HC}$ & 0.04176 & $\mathrm{C} 43$ & CT & -0.15148 & C69 & $\mathrm{CT}$ & -0.08669 \\
\hline H18 & $\mathrm{HC}$ & 0.04176 & H44 & $\mathrm{HC}$ & 0.04820 & $\mathrm{H} 70$ & $\mathrm{HC}$ & 0.08332 \\
\hline C19 & $\mathrm{CC}$ & 0.02379 & $\mathrm{H} 45$ & $\mathrm{HC}$ & 0.04840 & $\mathrm{H} 71$ & $\mathrm{HC}$ & 0.03810 \\
\hline $\mathrm{C} 20$ & $\mathrm{CD}$ & -0.11630 & $\mathrm{H} 46$ & $\mathrm{HC}$ & 0.03770 & $\mathrm{C} 72$ & $\mathrm{CT}$ & 0.03682 \\
\hline $\mathrm{H} 21$ & $\mathrm{HC}$ & 0.14289 & C47 & $\mathrm{CB}$ & -0.03452 & $\mathrm{H} 73$ & $\mathrm{HC}$ & -0.01265 \\
\hline $\mathrm{C} 22$ & $\mathrm{CC}$ & 0.02348 & C48 & CT & 0.02005 & H74 & $\mathrm{HC}$ & -0.06307 \\
\hline $\mathrm{N} 23$ & NO & -0.37815 & $\mathrm{H} 49$ & $\mathrm{HC}$ & 0.04630 & $\mathrm{C} 75$ & $\mathrm{C}$ & 0.74898 \\
\hline $\mathrm{C} 24$ & $\mathrm{CB}$ & 0.00421 & $\mathrm{H} 50$ & $\mathrm{HC}$ & 0.02166 & O76 & $\mathrm{O} 2$ & -0.82350 \\
\hline $\mathrm{C} 25$ & CT & -0.15121 & C51 & CT & -0.08751 & O77 & $\mathrm{O} 2$ & -0.81543 \\
\hline H26 & $\mathrm{HC}$ & 0.04541 & H52 & $\mathrm{HC}$ & 0.02275 & & & \\
\hline
\end{tabular}

${ }^{a}$ The nomenclature used for atom types and parameters is the same as in the Cornell et al. force field.

were employed, the integration time step was set at $1 \mathrm{fs}$ and all interactions were calculated at every step. No atoms had their positions fixed or their motions damped.

Quantum Mechanical Calculation of Energy Gaps. The theory used for calculating optical spectra from a fluctuating energy gap has been discussed in previous work. ${ }^{1}$ The reader is referred to this paper for details. The method requires the calculation of an energy gap correlation function from a time ordered trace of the fluctuation between ground and excited state for a chromophore at equilibrium. The energy gap sampling has to be sufficiently frequent to adequately sample the fastest oscillations of the energy gap. In the case of zinc-myoglobin this equated to a sampling frequency of once every 4 fs for the first $10 \mathrm{ps}$ of the MD production phase. 
The trajectory obtained from the 22 ps production run of the MD simulations was used to create time ordered structures for the QM calculations. The QM method employed in this study is implemented within the ab initio package Gaussian $98 .{ }^{15}$ The method consists of incorporating the point charges of the water and protein surrounding the $\mathrm{Zn}$-heme residue into the oneelectron Hamiltonian. This has the effect of polarizing the wave function with respect to the protein structure and solvent distribution.

Taking structures from the classical MD trajectories, single point CIS $^{20}$ calculations utilizing the $3-21 G^{* 17}$ basis set were performed to calculate the singlet energy gap between the ground and first two excited states for the zinc-heme unit at 4 fs intervals. The use of the $3-21 G^{*}$ basis set was to ensure that the calculations were tractable to the available computing resources. Trial CIS calculations showed that the orbitals involved in the excitation were centered purely on the zincheme moiety, and thus it was decided to treat the zinc-heme residue quantum mechanically while treating the rest of the protein and surrounding water classically, giving a QM system consisting of 77 atoms corresponding to 475 basis functions. In this way the need for link atoms was negated since the $\mathrm{Zn}$ heme residue is not formally bound to the protein matrix. The point charges of the classical system were included in the oneelectron Hamiltonian of the quantum element of the calculations.

A total of 2500 points representing the first 10 ps of the 22 ps production run were calculated for the $\mathrm{Zn}$-heme residue.

Calculation of the Steady-State Spectra. The absorption and emission spectra were generated from the time correlated energy gap fluctuations by utilizing the methodology found in Mercer et al. ${ }^{1}$ This methodology has recently been shown to work for protein based systems with Stokes shifts as large as $0.87 \mathrm{eV}^{3}$ and for solvated molecules with energy relaxation as small as $80 \mathrm{meV} .{ }^{1}$ Here we apply our methodology to a protein system with a peak shift of only $7 \mathrm{meV}$. Our previous calculations and arguments based on the nature of electron vibration coupling in proteins had shown that we could expect reasonable convergence of the Stokes shift after 5 to $10 \mathrm{ps}$. We chose our convergence criteria as previously with this methodology, namely that convergence was deemed to have occurred once the change in predicted Stokes shift was less than $10 \%$ for a doubling of the run length.

Calculation of the Energy Gap Dynamics (3PEPS). The energy gap autocorrelation function can be used to calculate nonlinear optical observables. The three pulse photon echo is a signal generated from a third order polarization requiring three electric field-matter interactions. Within the framework of the response function formalism, developed by Mukamel and coworkers, ${ }^{21}$ this third order polarization can be expressed in terms of a nonlinear response function which, by application of the cumulant expansion, can be described in terms of a line-shape function. The intensity of the echo signal at a photo detector is simply proportional to the square of the third order polarization integrated over the detection period. The integrated echo is a function of two variables, the time delay between the 1st and 2nd pulses, $\tau$ and the 2 nd and 3 rd pulses, T. A plot of the integrated echo as function $\tau$ at a fixed $\mathrm{T}$ has a maximum, and this maximum shifts toward zero $\tau$ delay as $\mathrm{T}$ increases. The 3PEPS function was simulated using the QMMM autocorrelation trace and by evaluating the required integrals numerically, taking into account the effect of the finite pulse duration.

Preparation of Zinc Substituted Myoglobin. Zinc protoporphyin IX (Zn-PP IX) substituted myoglobin was prepared according to previously published methods ${ }^{22-27}$ with minor modifications. The preparation is broadly divided into three stages consisting of iron extraction, $\mathrm{Zn}-\mathrm{PP}$ IX reconstitution and purification. Each of these stages is outlined below. The whole procedure was carried out at $4{ }^{\circ} \mathrm{C}$ with as little exposure to light as possible.

Stage 1 - Iron Heme Extraction Using the 2-Butanone Method. ${ }^{28,29}$ The method used for extracting the iron heme from the myoglobin protein matrix was based on the 2-butanone method of heme extraction. $0.15 \mathrm{~g}$ of iron myoglobin (SigmaAldrich) used without purification was dissolved in $30 \mathrm{~mL}$ of distilled water. The $\mathrm{pH}$ was lowered to 1.0 by adding $1 \mathrm{M} \mathrm{HCl}$ dropwise while stirring in order to denature the protein allowing the iron heme moiety to be extracted.

$100 \mathrm{~mL}$ of 2-butanone was added to the denatured myoglobin solution and shaken in a separating funnel. A hazy pale yellow aqueous layer (bottom) containing the protein separated from the dark brown iron-heme containing 2-butanone layer. The protein (apo-myoglobin) layer was shaken with a further two volumes of $100 \mathrm{~mL}$ of 2-butanone until the residual brown color disappeared.

The apo-myoglobin solution was transferred to a dialysis bag (Sigma-Aldrich) which had been prepared according to standard procedure. The solution was then exhaustibly dialyzed in $2 \times$ $1 \mathrm{~L}$ of $0.01 \mathrm{M}$ sodium bicarbonate solution, each lasting for 12 h. This was followed by dialysis with $1 \mathrm{~L} \times 0.01 \mathrm{M} \mathrm{pH} 7$ phosphate buffer for another $12 \mathrm{~h}$.

Stage 2 - Zn-Protoporphyrin IX Reconstitution. ${ }^{22,27}$ Approximately $6 \mathrm{mg}$ of $\mathrm{Zn}-\mathrm{PP}$ IX (Sigma-Aldrich) used without purification was dissolved in approximately 10 drops of $0.1 \mathrm{M} \mathrm{NaOH}$ and then added dropwise to a vigorously stirred solution of the apo-myoglobin from stage $1.1 \mathrm{M} \mathrm{HCl}$ was added as necessary to maintain a $\mathrm{pH}$ of 8.0-9.0 during the addition of the $\mathrm{Zn}-\mathrm{PP}$ IX to the apo-myoglobin. The solution was then stirred for $30 \mathrm{~min}$ before being neutralized with $1 \mathrm{M} \mathrm{HCl}$. Precipitate was then removed from the resulting solution by centrifuging.

Stage 3 - Purification. ${ }^{30}$ Sephadex CM C-50 ion exchange gel which had been prepared according to standard procedure was packed into a column and equilibrated with $0.01 \mathrm{M} \mathrm{pH} 7$ phosphate buffer. To this was applied the supernatant from stage 2. The Zn-PP IX substituted myoglobin was eluted with the phosphate buffer. Two clear bands were visible in the column: a fast moving first band (brown) and a slow moving second band (pink). The second band containing the $\mathrm{Zn}-\mathrm{PP} \mathrm{XI}$ substituted myoglobin band was collected.

Experimental Measurement of Spectra. Prior to measuring the absorption and emission spectra the Zn-PP IX substituted myoglobin solution was concentrated with a centrifugal filter device (Amicon Centricon Plus-20, Millipore). The absorption spectrum was then measured using a Shimadzu UV-1601 UVvisible spectrophotometer with a $1 \mathrm{~cm}$ path length cell. The optical density at $596 \mathrm{~nm}$ was measured to be 0.01 .

An emission spectrum and an excitation spectrum were measured using a Spex FluoroMax fluorometer using the same sample solution as the absorption spectrum.

Absorption peaks are observed at $596 \mathrm{~nm}, 553 \mathrm{~nm}, 428 \mathrm{~nm}$, $355 \mathrm{~nm}$ and $279 \mathrm{~nm}$, and the spectrum agrees well with previously published data. ${ }^{23,31}$ An intensity ratio of $\sim 1: 9$ for the $280 \mathrm{~nm}$ and $428 \mathrm{~nm}$ bands was observed, and this is generally accepted to indicate good sample purity. ${ }^{23,31,32}$ The emission spectrum contains two bands around $598 \mathrm{~nm}$ and 650 $\mathrm{nm}$, which again agrees well with previously published work. ${ }^{23}$ The experimental peak shift between the absorption and emission spectra is measured to be $7 \pm 0.5 \mathrm{meV}$. 
3PEPS Measurement. The three-pulse photon echo peak shift (3PEPS) measurements were performed with a home-built noncollinear optical parametric amplifier (NOPA), operating at $10 \mathrm{kHz}$ using a standard 3PEPS configuration. ${ }^{33}$ The NOPA was pumped by a commercial Coherent femtosecond oscillator and amplifier system (Coherent Vitesse-RegA).

The commercial femtosecond oscillator and amplifier system typically delivers $\sim 120 \mathrm{fs}$ long (fwhm), $\sim 4 \mu \mathrm{J}$ pulses centered at $800 \mathrm{~nm}$. This so-called fundamental beam was used to generate a single-pass noncollinearly phase-matched optical parametric amplification. The low repetition $(1 \mathrm{kHz})$ rate and high pulse energy $(\sim 500 \mu \mathrm{J})$ versions are extensively reported in the literature. ${ }^{34} \mathrm{~A}$ brief description of our high repetition rate, low pulse energy set up is as follows. The fundamental beam was split into a ratio of $80: 20,3.2 \mu \mathrm{J}$ going into the pump arm and $0.8 \mu \mathrm{J}$ into the continuum arm. In the pump arm, the fundamental beam was frequency doubled in a $1 \mathrm{~mm}$ thick $\beta$-barium borate (BBO) crystal to generate the second harmonic (SH) beam centered at $400 \mathrm{~nm}$. In the continuum arm, the beam was focused into a $1 \mathrm{~mm}$ thick sapphire disk, generating the white-light continuum. Finally the SH beam and continuum were combined in a second $1 \mathrm{~mm}$ thick BBO crystal, producing $\sim 20$ fs pulses (after the pulse compression) that were tunable from $500 \mathrm{~nm}$ to $650 \mathrm{~nm}$. Pulse energies of up to $60 \mathrm{~nJ}$ were readily achieved.

The 3PEPS configuration is based largely on the design previously reported by the Fleming group. ${ }^{35}$ Briefly, the beam from the NOPA was split into three roughly equal energy pulses and sent along different paths. Of those, two travel over computer controlled variable delay stages. The three beams, forming an equilateral triangle geometry at the $20 \mathrm{~cm}$ singlet focusing lens, then recombine at the sample. The experimentally variable parameters are the two time delays facilitated by the delay stages. The first delay is called the coherence period and is the delay between the first and second pulses. During this period the superposition state of the ground and excited state created by the first pulse evolves. The second pulse then creates the population state either in the ground or excited state. This state evolves until the arrival of the third beam. This period is controlled by the second delay time, the population period. The arrival of the third pulse creates the coherence again. If the phase during the second coherence period is the complex conjugate of the first, the sample radiates a coherent echo field, sometime after the third pulse. The echo signal was detected and integrated using a purpose built silicon photodetector, preamp setup using a lock-in amplifier. The integrated echo was measured as the function of the first coherence delay at a fixed second delay. The echo peak shift is the first coherence delay time at which the integrated echo profile as function of the first time delay is at a maximum. The echo peak shift function or 3PEPS is the change in the echo peak shift as function of the second delay time, the population period.

The protein sample, prepared as outlined above, was sandwiched between two quartz windows and placed in the custommade sample holder. A $250 \mu \mathrm{m}$-thick sample had an optical density of $\sim 0.2$ at $596 \mathrm{~nm}$. The sample was spun during the measurement such as to prevent the build-up of the triplet state and multiple excitations.

\section{Results and Discussion}

The evaluation of the electronic energy gaps yielded two nearly degenerate transitions. This is in agreement with a body of literature that suggests the experimentally observed peak at $596 \mathrm{~nm}$, which is the lowest electronic transition (i.e., $(0,0)$

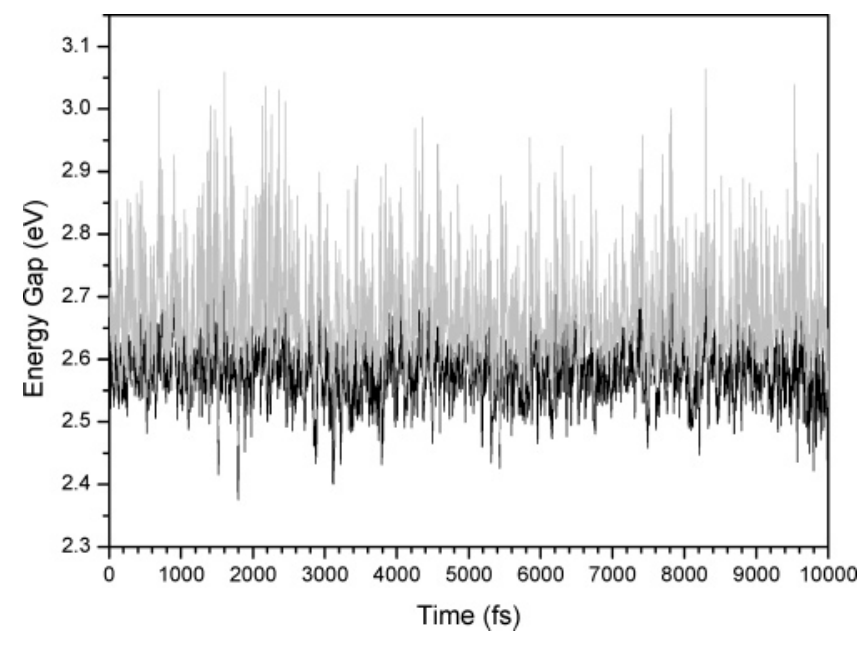

Figure 1. Calculated ground to excited-state energy gap trace against time for zinc-myoglobin. The first two excited states are shown and are labeled according to their energy. The lowest energy transition is shown in black, with the second lowest in gray.

transition), is a convolution of two nearly degenerate, orthogonal states, known as $\mathrm{Q}_{x}$ and $\mathrm{Q}_{y}$ bands. ${ }^{36}$

The QM/MM calculation delivers the time ordered trace of the fluctuation between ground and excited state for the chromophore at equilibrium. In zinc-myoglobin there are two such traces to consider, due to the existence of two excited states close in energy.

The near degeneracy of the two excited states is troublesome because the magnitude of the fluctuation of the energy gaps is found to be bigger than the difference in energy between the two excited states. This causes difficulties in labeling the states. In short, this is because each QM calculation corresponding to a particular structural configuration in the MD trajectory returns energies and the transition dipole moments of the two transitions (hence oscillator strength) only. It says nothing about how those two states are related to the two states present at other times. It is not entirely obvious how one should label the states such that the spectroscopy would be best represented. To deal with this problem, three types of assignments are made by the energy, oscillator strength and the transition dipole moment (TDM) direction. Once assigned, the energy gap fluctuations are reconstructed for every assignment method. Assignment by energy most closely agreed with experiment, and these results are shown in Figure 1, with the corresponding absorption and emission spectra shown in Figure 2. The absorption and emission spectra are the sum of spectra due to the two excited states that are weighted by the calculated oscillator strength.

The absorption width and the peak shift are recovered within a factor of 2. In all our previous work the method of electronvibration coupling calculation has reproduced the absorption width and the peak shift to within $20 \%$ accuracy in proteins with very large $(\sim 0.87 \mathrm{eV})^{3}$ as well as small $(0.080 \mathrm{eV})^{1}$ energy relaxation (the peak shift).

The factor of 2 discrepancy in the peak shift is somewhat surprising, given our previous level of accuracy. The discrepancies are also observed in the calculated and measured 3PEPS. Figure 3 shows the 3PEPS trace measured at $596 \mathrm{~nm}$ laser wavelength. For comparison, the calculated 3PEPS traces are also shown, using the same energy gap traces used to generate the steady-state spectra. The two calculated 3PEPS traces correspond to two excited states. Since there is no simple way to treat two coupled transitions within our current theoretical framework, the calculated 3PEPS traces shown are two indi- 


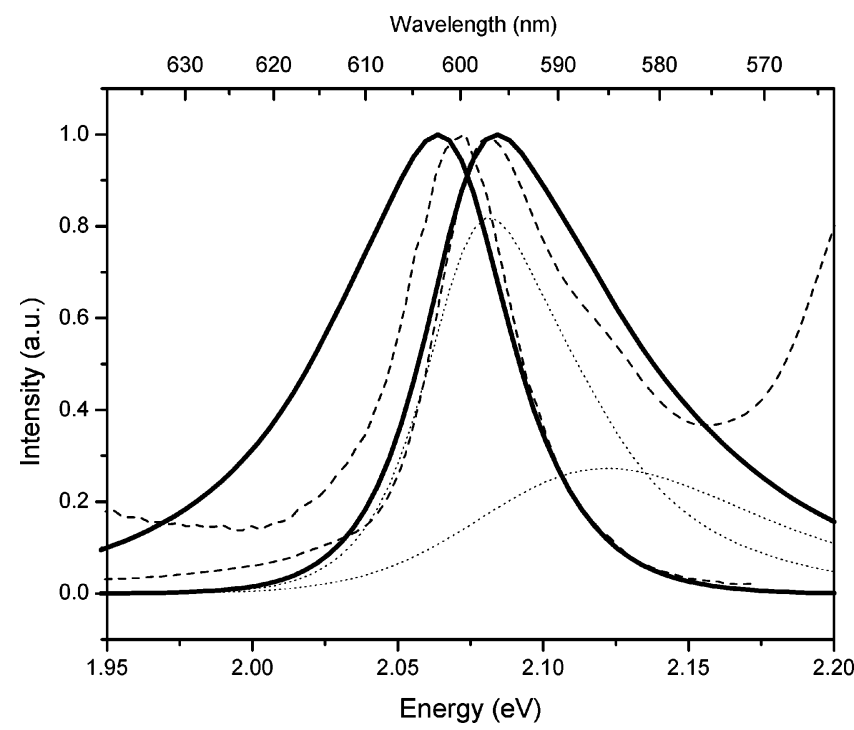

Figure 2. Calculated (solid line) and experimental (dashed line) absorption and emission spectra for ZnP-MB. The calculated individual spectra corresponding to the first and the second excited states are shown in dotted lines. The overall absorption spectra are the sum of the spectra of the excited states weighed according to the calculated oscillator strengths.

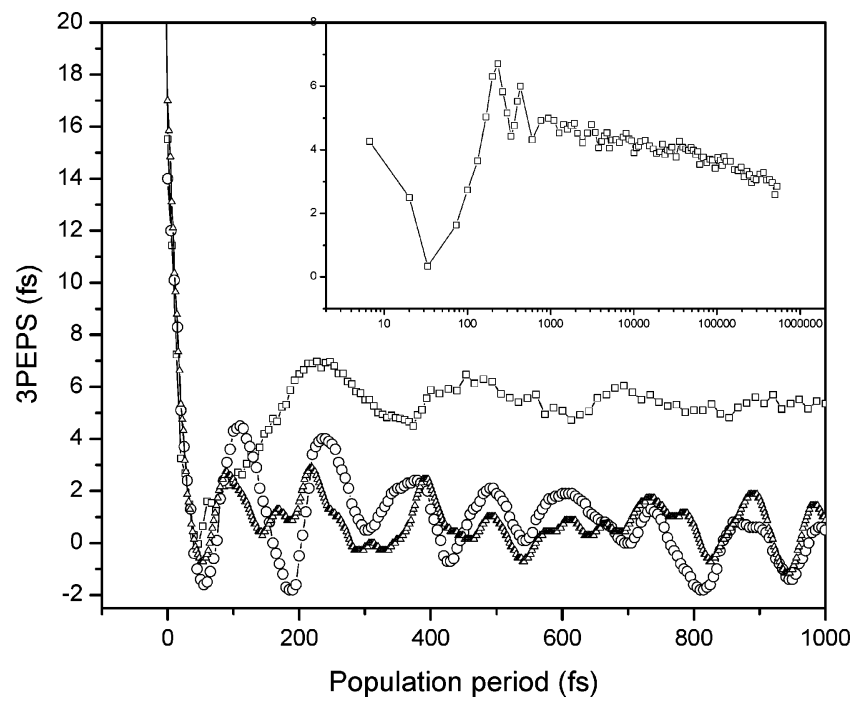

Figure 3. Calculated (circle and triangle) and measured (square and inset) zinc myoglobin 3PEPS are compared. There are two calculated 3PEPS traces, corresponding to two excited states. The ultrafast initial decay is well reproduced in the calculation. However, the long-time offset (see inset) and oscillatory features observed in the measured 3PEPS do not agree well (see text).

vidual 3PEPS traces calculated as if the two states were assumed to be independent of one another. This is in contrast to our previous attempt on chlorophyll a which has a single excitation energy and shows a good match. ${ }^{37}$

Figure $4 \mathrm{~b}$ shows the plot of the auto-correlation function of the angle between the two TDMs corresponding to excitation to the first two excited states labeled by the energy. It is evident that the TDM angle does not span random values during the course of the MD simulation, in which case the TDM angle auto-correlation function would show a rapid drop to a value of zero and remain at zero. The oscillation seen in Figure $4 \mathrm{~b}$ is indicative of some correlated behavior.

Given the fact that the two electronic transitions are close in energy, one might expect to see effects of electronic coherence. $^{38,39}$ To test this idea, using the calculated TDM vectors
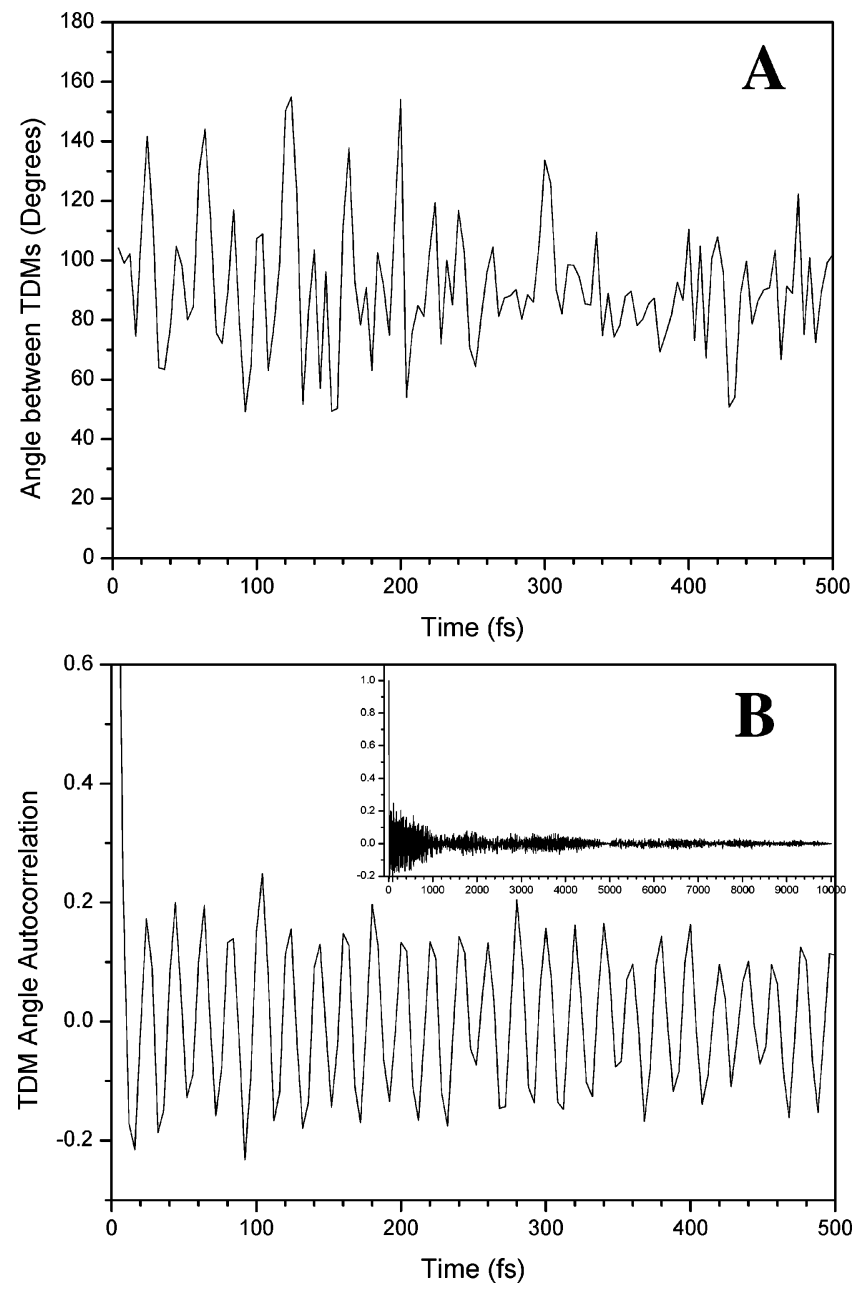

Figure 4. Autocorrelation (B) of the TDM angle (A) between the two nearly degenerate $\mathrm{ZnP}-\mathrm{MB}$ excited states. A correlation (B) between the TDM angles (A) is clearly visible implying that the TDM angle between the two excited states does not span random values but instead oscillates in a correlated fashion suggesting a coupling of the two states. Inset shows full $10 \mathrm{ps}$ time window.

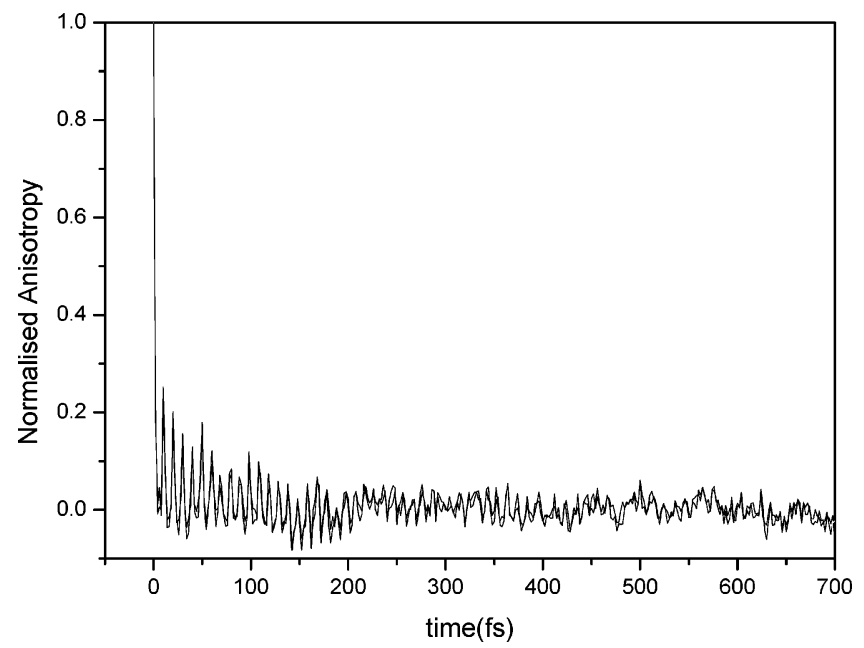

Figure 5. Calculated anisotropy when electronic coherence is assumed (black) or not assumed (grey). No significant difference is observed. However, oscillation is indicative of nonrandom reorientation of the time dependent TDM. This suggests that the two states are adiabatically coupled.

for the two transitions, time dependent anisotropy is simulated when either coherence or incoherence is assumed. As Figure 5 shows, there is no significant difference in the anisotropy 
dynamics, indicating that electronic coherence is not inherent in the system. However, it is noted that an oscillation is clearly seen. Because the molecule does not tumble in the time scale of the MD simulation, the decay in anisotropy must be due to rapid reorientation of the time dependent TDM and the oscillation therefore implies nonrandom reorientation of the TDM.

Figure 5 strongly suggests that the two electronic states interact as their dynamics are not independent of each other, in which case they might be said to be adiabatically coupled. ${ }^{40}$ This has an obvious implication for calculating the optical observables. Specifically, it is not obvious how to account for the adiabaticity in the current theoretical framework. The discrepancies seen, both in the calculated steady-state spectra and the 3PEPS function, may therefore be due to not properly accounting for the adiabaticity. Despite the fact that the effect of adiabaticity is evident, without a theoretical framework that includes this coupling implicitly, it is difficult to simulate the effect of adiabaticity on the 3PEPS signal. As such, other factors which could contribute to the 3PEPS signal may merit discussion. A number of works ${ }^{41,42}$ have shown that internal conversion time scales may give rise to a decay or rise of the 3PEPS, when excited-state absorption contributes to the 3PEPS signal. Others have indeed shown that related heme proteins such as cytochrome $c$ undergo complex internal conversion dynamics. ${ }^{43,44}$ One may also need to consider the effect of ligand dissociation and recombination of the proximal histidine sidechain. ${ }^{45,46}$ A topic that the classical MD simulation used in this study was not capable of reproducing but an area worth investigating, in future work, with a coupled potential QMMM/ MD approach in which the formation and subsequent breaking of the $\mathrm{N}-\mathrm{Zn}$ proximal histidine bond on a femtosecond time scale could be implicitly included. The time scales of such processes may be responsible for the rise and decay of the 3PEPS in the first $100 \mathrm{fs}$. Such dynamics will contribute to the 3PEPS signal in a complicated way, especially in the first hundreds of femtoseconds. Again, the modeling that incorporates these effects is beyond the scope of this paper, given the fact that one still needs to account for the adiabaticity. Nevertheless lessons can be drawn from the comparison between the calculations and the experiments. The comparison is relevant because there is a good qualitative agreement despite the fact the aforementioned effects are not explicitly modeled. This indicates that a significant portion of the physics is wellrepresented in the QM/MM method.

Although we have assumed no coupling between the two states in the generation of the steady-state spectra the main features are successfully reproduced. This suggests that the QM/ MM method gets the coupling strength and the dynamics roughly right. We have also simulated separate 3PEPS functions for the two energy gap traces. Immediately obvious from Figure 3 is that there is a good agreement in the ultrafast decay in the first $50 \mathrm{fs}$. However, the long-time offset and oscillatory features are not very well reproduced in either of the two calculated traces.

Physical interpretations usually given for the initial rapid decay are that they are due to the inertial motion of the solvent ${ }^{47}$ or inertial motions inside the protein matrix ${ }^{48,49}$ that are coupled to the energy level or destructive interference of the impulse excitation of vibration by short laser pulses. ${ }^{50}$ Generally, in condensed phase, these sorts of ultrafast dynamics account for the majority of the amplitude and the 3PEPS of ZnP-MB does not seem to be an exception. A good agreement in the ultrafast

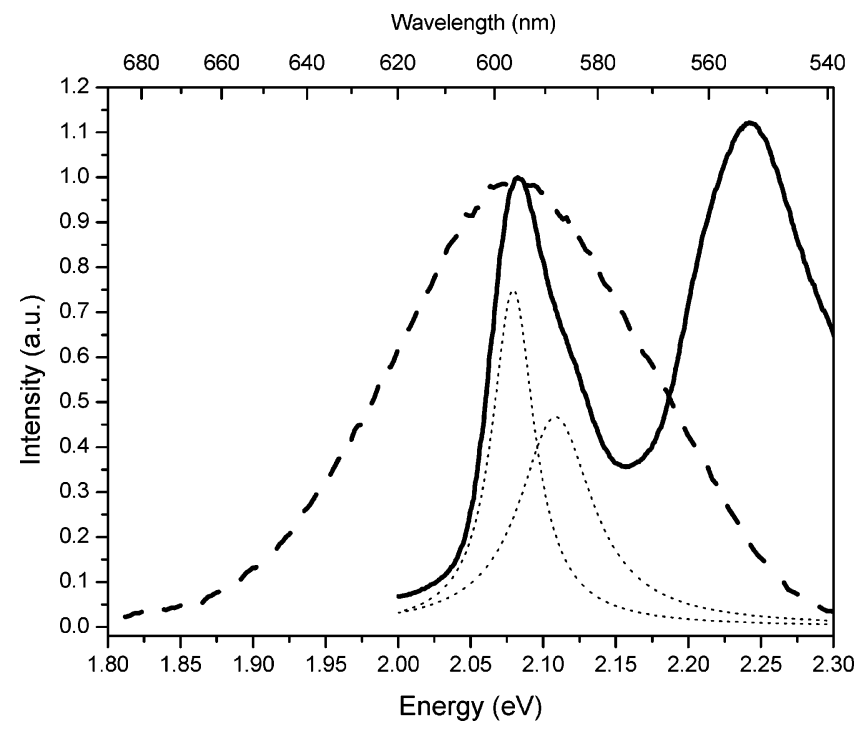

Figure 6. The $596 \mathrm{~nm}$ laser pulse spectrum (dashed) is overlaid with $\mathrm{ZnP}-\mathrm{MB}$ absorption spectrum (solid). Dotted lines show Lorentzian band deconvolution of the $\mathrm{Q}(0,0)$ band representing two electronic states that are separated by $\sim 20 \mathrm{meV}(\sim 0.80 \mathrm{kT})$.

dynamics shows that fast fluctuations are well represented in the calculation.

On the other hand, the slower dynamics that are clearly observed in the experiment (long time offset) reflect inhomogeneity. This inhomogeneity is presumably the residual memory of each of the initially excited electronic states. The optical pulse is short enough to excite both states simultaneously (Figure 6), and for the 3PEPS signal to reach zero, both states would have had to have lost their initial identity fully. This long term inhomogeneity therefore suggests that the two states do not completely mix on this time scale. Indeed the projection of the angle between the two dipoles shown in Figure 4 suggests that the two states retain some independence for a few picoseconds. On the other hand, it might reflect genuine slow time scale dynamics whereby the memory of the chromophore transition frequency is made to last due to being enclosed inside the protein matrix. Certainly, the time scales seen in the measurements are not inconsistent with those reported in the literature that sought to find out the effect of being "solvated" by a protein bulk as opposed to being exposed directly to small solvent molecules. $^{47,48,51}$

Recently Prall et al. ${ }^{52}$ have shown that two-color photon echo peak shift spectroscopy can probe the electronic coupling in a phthalocyanine homodimer. The existence of a two-color peak shift revealed the existence of correlation between the two dipole-allowed states. The same technique applied to zinc myoglobin may reveal experimentally the correlation of the two excited states.

Another obvious discrepancy is the frequencies of the oscillatory modulations on the 3PEPS trace. Linear prediction singular value decomposition analysis on the measured 3PEPS yields two significant oscillations of 158 and $141 \mathrm{~cm}^{-1}$ with 229 and 44 fs damping times, respectively. In the calculated 3PEPS, for state 1 , there are two notable oscillations at 268 and $98 \mathrm{~cm}^{-1}$ with $\sim 200$ and 390 fs damping time, respectively. For state 2, two prominent oscillations of $254 \mathrm{~cm}^{-1}$ and 154 $\mathrm{cm}^{-1}$ are observed with 125 and 188 fs damping times, respectively.

There are a number of mechanisms by which the 3PEPS trace can show oscillatory modulation. ${ }^{53}$ A vibration which may be inter- or intramolecular in origin could couple strongly to the 
energy level. Such strong coupling could also produce oscillations at the frequency of the vibration. A far IR measurement on the iron heme compound has reported a vibration at 158 $\mathrm{cm}^{-1}$ which was assigned to ethane twisting. ${ }^{54}$

\section{Conclusions}

The QM/MM methodology has been applied to calculate the electron-vibration coupling inside zinc myoglobin whose energy relaxation is $\sim 0.007 \mathrm{eV}$. A good qualitative agreement in the absorption width and the peak shift was observed. This strongly suggests that the methodology performs well in estimating the magnitude of electron-vibration coupling. As for the dynamics, a time dependent optical observable, namely 3PEPS was measured and calculated. The comparison reveals that fast fluctuations are well represented in the QM/MM calculation as demonstrated by the good agreement in the ultrafast initial decay of the 3PEPS traces.

Previous calculations that were performed on proteins with energy relaxations as large as $0.87 \mathrm{eV}$ and as small as 0.080 $\mathrm{eV}$ demonstrate the versatility of the QM/MM methodology in accounting for electron-vibration coupling which may be fundamental to understanding chemical dynamics in condensed phases. Other features such as the rise and decay in the 3PEPS in the first few hundreds fs and the oscillatory features are not very well represented. The discrepancies seen in both steadystate spectra and 3PEPS may be due to near 2-fold degeneracy which may be adiabatically coupled. In the 3PEPS signal, there may be other additional processes such as internal conversion and/or ligand dissociation and recombination that may contribute, especially within a couple of hundred fs. Currently there is no provision in the theoretical framework that can account for the effect of adiabaticity. Such deficiencies will form the basis of future work aimed at improving our QM/MM methodology.

Acknowledgment. The authors would like to thank the Engineering and Physical Sciences Research Council (EPSRC) [Grant Number GR/M46730] for funding this research. These two authors contributed equally to this work (B.M.C. and R.C.W.).

\section{References and Notes} 279.

(1) Warshel, A.; Parson, W. W. Annu. Rev. Phys. Chem. 1991, 42,

(2) Mercer, I. P.; Gould, I. R.; Klug, D. R. J. Phys. Chem. B 1999, 103,7720

(3) Walker, R. C.; de Souza, M. M.; Mercer, I. P.; Gould, I. R.; Klug, D. R. J. Phys. Chem. B 2002, 106, 11658.

(4) Case, D. A.; Pearlman, D. A.; Caldwell, J. W.; Cheatham, T. E., III.; Ross, W. S.; Simmerling, C. L.; Darden, T. A.; Merz, K. M.; Stanton, R. V.; Cheng, A. L.; Vincent, J. J.; Crowley, M.; Tsui, V.; Radmer, R. J.; Duan, Y.; Pitera, J.; Massova, I.; Seibel, G. L.; Singh, U. C.; Weiner, P. K.; Kollman, P. A. AMBER 6 University of California, San Francisco, 1999.

(5) Maurus, R.; Overall, C. M.; Bogumil, R.; Luo, Y.; Mauk, A. G.; Smith, M.; Brayer, G. D. Biochim. Biophys. Acta-Protein Struct. Mol. Enzymol. 1997, 1341, 1.

(6) Schafmeister, C. E.; Ross, W. S.; Romanovski, V. LEaP; University of San Francisco, 1995.

(7) Bhattacharya, S.; Sukits, S. F.; MacLaughlin, K. L.; Lecomte, J. T. J. Biophys. J. 1997, 73, 3230.

(8) Bashford, D.; Case, D. A.; Dalvit, C.; Tennant, L.; Wright, P. E. Biochemistry 1993, 32, 8045.

(9) Cornell, W. D.; Cieplak, P.; Bayly, C. I.; Gould, I. R.; Merz, K. M.; Ferguson, D. M.; Spellmeyer, D. C.; Fox, T.; Caldwell, J. W.; Kollman, P. A. J. Am. Chem. Soc. 1995, 117, 5179.

(10) Giammona, D. A. Ph.D. Thesis, University of California, Davis, 1984.

(11) Jorgensen, W. L.; Chandrasekhar, J.; Madura, J. D.; Impey, R. W.; Klein, M. L. J. Chem. Phys. 1983, 79, 926.

(12) Bayly, C. I.; Cieplak, P.; Cornell, W. D.; Kollman, P. A. J. Phys. Chem. 1993, 97, 10269 .
(13) Roothaan, C. C. J. Rev. Modern Phys. 1951, 23, 69. 2657.

(15) Frisch, M. J.; Trucks, G. W.; Schlegel, H. B.; Scuseria, G. E.; Robb M. A.; Cheeseman, J. R.; Zakrzewski, V. G.; Montgomery Jr., J. A.; Stratmann, R. E.; Burant, J. C.; Dapprich, S.; Millam, J. M.; Daniels, A. D.; Kudin, K. N.; Strain, M. C.; Farkas, O.; Tomasi, J.; Barone, V.; Cossi, M.; Cammi, R.; Mennucci, B.; Pomelli, C.; Adamo, C.; Clifford, S.; Ochterski, J.; Petersson, G. A.; Ayala, P. Y.; Cui, Q.; Morokuma, K.; Salvador, P.; Dannenberg, J. J.; Malick, D. K.; Rabuck, A. D.; Raghavachari, K.; Foresman, J. B.; Cioslowski, J.; Ortiz, J. V.; Baboul, A. G.; Stefanov, B. B.; Liu, G.; Liashenko, A.; Piskorz, P.; Komaromi, I.; Gomperts, R.; Martin, R. L.; Fox, D. J.; Keith, T.; Al-Laham, M. A.; Peng, C. Y.; Nanayakkara, A.; Challacombe, M.; Gill, P. M. W.; Johnson, B.; Chen, W.; Wong, M. W.; Andres, J. L.; Gonzalez, C.; Head-Gordon, M.; Replogle, E. S.; Pople, J. A. Gaussian 98 (Revision A. 11); Gaussian, Inc., Pittsburgh, P A, 2001.

(16) Schafer, A.; Horn, H.; Ahlrichs, R. J. Chem. Phys. 1992, 97, 2571.

(17) Binkley, J. S.; Pople, J. A.; Herhe, W. J. J. Am. Chem. Soc. 1980, 102,939 .

(18) Berendsen, H. J.; Postma, J. P. M.; Van Gunsteren, W. F.; DiNola, A.; Haak, J. R. J. Chem. Phys. 1984, 81, 3684.

(19) Essmann, U.; Perera, L.; Berkowitz, M. L.; Darden, T.; Lee, H.; Pedersen, L. G. J. Chem. Phys. 1995, 103, 8577.

(20) Foresman, J. B.; Headgordon, M.; Pople, J. A.; Frisch, M. J. J. Phys. Chem. 1992, 96, 135.

(21) Mukamel, S. Principles of Optical Nonlinear Spectroscopy, 1st ed.; Oxford University Press: Oxford, New York, 1995.

(22) Ascoli, F.; Fannelli, M. R. R.; Antonini, E. Method Enzymol. 1981, 76.

(23) Papp, S.; Vanderkooi, J. M.; Owen, C. S.; Holtom, G. R.; Philips, C. M. Biophys. J. 1990, 58, 177

(24) Rothgeb, T. M.; Gurd, F. R. N. Method Enzymol. 1978, 52, 473.

(25) Shosheva, A. C.; Christova, P. K.; Atanasov, B. P. Biochem. Biophys. Acta 1988, 957, 202.

(26) Shosheva, A. C.; Devedjiev, Y. D.; Atanasov, B. P. Studia Biophys. 1989, $129,77$.

(27) Barboy, N.; Feitelson, J. Biochemistry 1989, 28, 5450.

(28) Teal, F. W. Biochem. Biophys. Acta 1959, 35, 543.

(29) Yonetani, T. J. J. Biol. Chem. 1967, 242, 5008

(30) Barboy, N.; Feitelson, J. Biochemistry 1987, 26, 3240.

(31) Aono, S.; Nemoto, S.; Okura, I. Bull. Chem. Soc. Jpn. 1992, 65 , 591.

(32) Tsukahara, K.; Asami, S.; Okada, M.; Sakurai, T. Bull. Chem. Soc Jpn. 1994, 67, 421.

(33) Cho, B. M. Protein Dynamics Measured By Nonlinear Spectroscopy, Department of Chemistry, Imperial College, Ph.D. Thesis 2003.

(34) Cerullo, G.; De Silvestri, S. Rev. Sci. Instrum. 2003, 74, 1.

(35) Joo, T. H.; Jia, Y. W.; Yu, J. Y.; Lang, M. J.; Fleming, G. R. J. Chem. Phys. 1996, 104, 6089.

(36) Gouterman, M. Optical Spectra and Electronic Structure of Porphyrins and Related Rings; Academic Press: New York, 1978; Vol. III.

(37) Mercer, I. P.; Abend, S.; Gould, I. R.; Klug, R. D. Quantum Mechanical/Molecular Mechanical Approach to Solvation Dyanmics Tested by Three Pulse Photon Echo Measurement; Ultrafast Phenomena XI, 1998; Germany.

(38) Wynne, K.; Hochstrasser, R. M. Chem. Phys. 1993, 171, 179.

(39) Galli, C.; Wynne, K.; Lecours, S. M.; Therien, M. J.; Hochstrasser, R. M. Chem. Phys. Lett. 1993, 206, 493.

(40) Wynne, K.; Hochstrasser, R. M. Coherence and adiabaticity in ultrafast electron transfer. In Advances in Chemical Physics; Jortner, J., Bixon, M., Eds., 1999; Vol. 107, p 263.

(41) Yang, M.; Ohta, K.; Fleming, G. R. J. Chem. Phys. 1999, 110 10243

(42) Xu, Q. H.; Scholes, G. D.; Yang, M.; Fleming, G. R. J. Phys. Chem. A 1999, 103, 10348.

(43) Wang, W.; Ye, X.; Demidov, A. A.; Rosca, F.; Sjodin, T.; Cao,

W. X.; Sheeran, M.; Champion, P. M. J. Phys. Chem. B 2000, 104, 10789.

(44) Jimenez, R.; Romesberg, F. E. J. Phys. Chem. B 2002, 106, 9172.

(45) Lampa-Pastirk, S.; Lafuente, R. C.; Beck, W. F. J. Phys. Chem. B 2004, 108, 12602

(46) Cao, W. X.; Ye, X.; Sjodin, T.; Christian, J. F.; Demidov, A. A.; Berezhna, S.; Wang, W.; Barrick, D.; Sage, J. T.; Champion, P. M. Biochemistry 2004, 43, 11109.

(47) Jordanides, X. J.; Lang, M. J.; Song, X. Y.; Fleming, G. R. J. Phys. Chem. B 1999, 103, 7995.

(48) Jimenez, R.; Case, D. A.; Romesberg, F. E. J. Phys. Chem. B 2002, 106,1090

(49) Kennis, J. T. M.; Larsen, D. S.; Ohta, K.; Facciotti, M. T.; Glaeser, R. M.; Fleming, G. R. J. Phys. Chem. B 2002, 106, 6067.

(50) deBoeij, W. P.; Pshenichnikov, M. S.; Wiersma, D. A. J. Phys. Chem. 1996, 100, 11806. 
(51) Homoelle, B. J.; Edington, M. D.; Diffey, W. M.; Beck, W. F. J. Phys. Chem. B 1998, 102, 3044.

(52) Prall, B. S.; Parkinson, D. Y.; Yang, M.; Ishikawa, N.; Fleming, G. R. J. Chem. Phys. 2004, 120, 2537.
(53) Ohta, K.; Larsen, D. S.; Yang, M.; Fleming, G. R. J. Chem. Phys. 2001, 114, 8020.

(54) Klug, D. D.; Zgierski, M. Z.; Tse, J. S.; Liu, Z.; Kincaid, J. R.; Czarnecki, K.; Hemley, R. J. Proc. Natl. Acad. Sci. U.S.A. 2002, 99, 12526. 